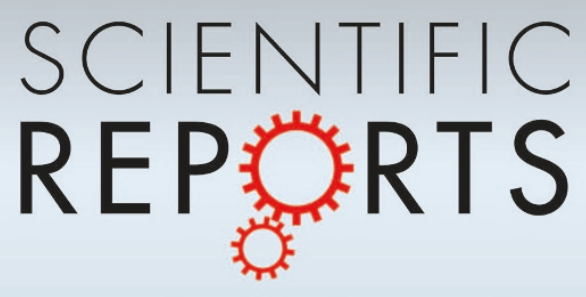

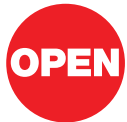

SUBJECT AREAS: BIOMECHANICS

MECHANICAL ENGINEERING ANIMAL PHYSIOLOGY ENTOMOLOGY

Received

4 October 2012

Accepted

17 December 2012

Published

22 January 2013

Correspondence and requests for materials should be addressed to

J.O.W. (jwolff@ zoologie.uni-kiel.de)

\section{Radial arrangement of Janus-like setae permits friction control in spiders}

\author{
Jonas O. Wolff \& Stanislav N. Gorb
}

Zoological Institute: Functional Morphology and Biomechanics, Kiel University, Am Botanischen Garten 1-9, D-241 18 Kiel, Germany.

Dynamic attachment is the key to move on steep surfaces, with mechanisms being still not well understood. The hunting spider Cupiennius salei (Arachnida, Ctenidae) possesses hairy attachment pads (claw tufts) at its distal legs, consisting of directional branched setae. The morphological investigation revealed that adhesive setae are arranged in a radial manner within the distal tarsus. Friction of claw tufts on smooth glass was measured to reveal the functional effect of seta arrangement within the pad. Measurements revealed frictional anisotropy in both longitudinal and transversal directions. Contact behaviour of adhesive setae was investigated in a reflection interference contrast microscope (RICM). Observations on living spiders showed, that only a small part of the hairy pads is in contact at the same time. Thus the direction of frictional forces is depending on leg placement and rotation. This may aid controlling the attachment to the substrate.

M any animals are capable of moving on smooth walls or ceiling with ease due to the presence of adhesive pads on the ventral surface of their extremities. High adhesion can be achieved by those pads consisting of a dense array of numerous hairs terminating in small thin flexible plate like extensions called spatulae $^{1}$. Hairy attachment devices become a focus of interest in morphological, biomechanical, and the interdisciplinary biomimetic research ${ }^{2-4}$. Spiders, possessing hierarchical hairy "dry" adhesive system that is convergently evolved to that of gecko, are overlooked in recent biomechanical research. Nevertheless, spiders exhibit very interesting morphological features, such as for example strongly anisotropic distribution of spatulae on the differently shaped distal portion of each seta called lamella ${ }^{5}$. Furthermore, the functional mechanism of spider pads is presumably based on dry adhesion ${ }^{6,7}$, although it is influenced by ambient humidity ${ }^{8}$, and secretions occasionally may occur ${ }^{9,10}$.

Hairy adhesive pads are reported from diverse wandering spiders of the dionychan (two-clawed) clade ${ }^{11}$, but also occur in some mygalomorphs, lycosoids, dysderoids, palpimanoids and species of the agelenoid family Desidae $^{12}$. In web building taxa, especially the Orbiculariae, they are absent. Thus, their occurrence may be correlated with a free hunting lifestyle. In spiders, arrays of adhesive (tenent) setae are termed scopula ${ }^{13}$. Those partly occur on the ventral parts of the tibia, metatarsus and tarsus. Distal most pretarsal scopulae (claw tufts), located underneath the tarsal claws, are of major importance for locomotion. Their setal density is usually very high $\left(>2000 \mathrm{~mm}^{-2}\right)^{5}$.

Tenent setae have previously been shown to have a high adhesive performance ${ }^{7}$. Attractive forces are gained by a close contact between seta and substrate through the small sized thin and flexible spatulae. Thus, it was hypothesized that short-range intermolecular van der Waals forces mainly contribute to the adhesion mechanism of spider spatula $^{7}$. These forces should also lead to a strong friction resisting shear along the substrate, when spatulae are in contact. This would be important to provide a secure grip especially, when climbing vertically, but might also be important, when walking horizontally on smooth or micro rough surfaces, where friction, required for propulsion generation, can not be produced by the claws. Since spatulae only cover one side of the tape-like seta, friction is highly dependent on shear direction ${ }^{14}$. However, these force measurements were performed only with tarsal and metatarsal scopulae. Interestingly, in both studied species higher friction forces were recorded when leg segments were moved in the distal direction. This is rather surprising result, which is opposite to what had been previously hypothesized for claw tufts by Hill ${ }^{15}$ and what has been found in hairy attachment pads of geckos ${ }^{16}$ and insects ${ }^{17-19}$.

Here we present the first study of the frictional behaviour of the spider claw tufts. Following questions were asked: 1. Is there an anisotropy of frictional properties in the claw tuft? 2. Does frictional behaviour of the claw tuft differ from that previously found in tarsal and metatarsal scopulae? 3. Is there a difference in friction (and adhesion) between freshly ablated and air dried samples? 4. Is there a correlation between both apparent and real claw tuft areas and the frictional forces generated during sliding? 5. Is there a lateral frictional anisotropy between pro- and retrolateral lobes of the claw tuft? 


\section{Results}

Behavioural observations. Spiders were usually resting vertically with the prosoma facing down. Adhering on the smooth vertical glass surfaces of their container was no difficulty for these big spiders (weight about $4 \mathrm{~g}$ ). Even shaking the glass did not lead to a displacement of the spider.

When walking on smooth planar surfaces, spiders only use the claw tufts in contact, and the tarsus is hold in an angle of about $30^{\circ}$ to the substrate. Furthermore, the tarsus is concavely curved on its ventral side. Thus, the tarsal scopula is not planar and, presumably, can not achieve large contact area on plane surfaces. Tarsal scopula was only occasionally observed in contact, when spiders crossed edges or curved surfaces during walking. Similar configuration was observed in some resting animals within the glass container, when the anterior leg was outstretched. In those cases, the leg was slightly rotated so that lateral parts of the tarsal scopula came in contact. In walking spiders, their claws are usually retracted and slightly elevated above the substrate.

Morphology. The general structure of claw tufts in C. $s a l e i^{2,5}$ (Fig. 1A) is similar to those first described in sparassids ${ }^{13}$, philodromids ${ }^{20}$, and salticids ${ }^{15}$. Lamelliform setae (Fig. 1C) stand in a dense array (about 2400 setae $\cdot \mathrm{mm}^{-2}$ ) and bear distally branching microtrichia (setules) terminating with spatulae on their substrate facing sides. These are responsible for high friction and adhesion of the claw tuft ${ }^{7,14}$. The adhesive sides are orientated proximally in the distal and middle part of the claw tuft, whereas they are more turned towards the middle in the lateral and proximal parts of it (Fig. 1B).

The claw tuft consists of two lobes, the pro- and the retrolateral ones, each originating from a cuticular plate (tenent plate). Both cuticular plates together with the tarsal claws form the pretarsus (Fig. 1C). The pretarsus is movable relative to the tarsus and can
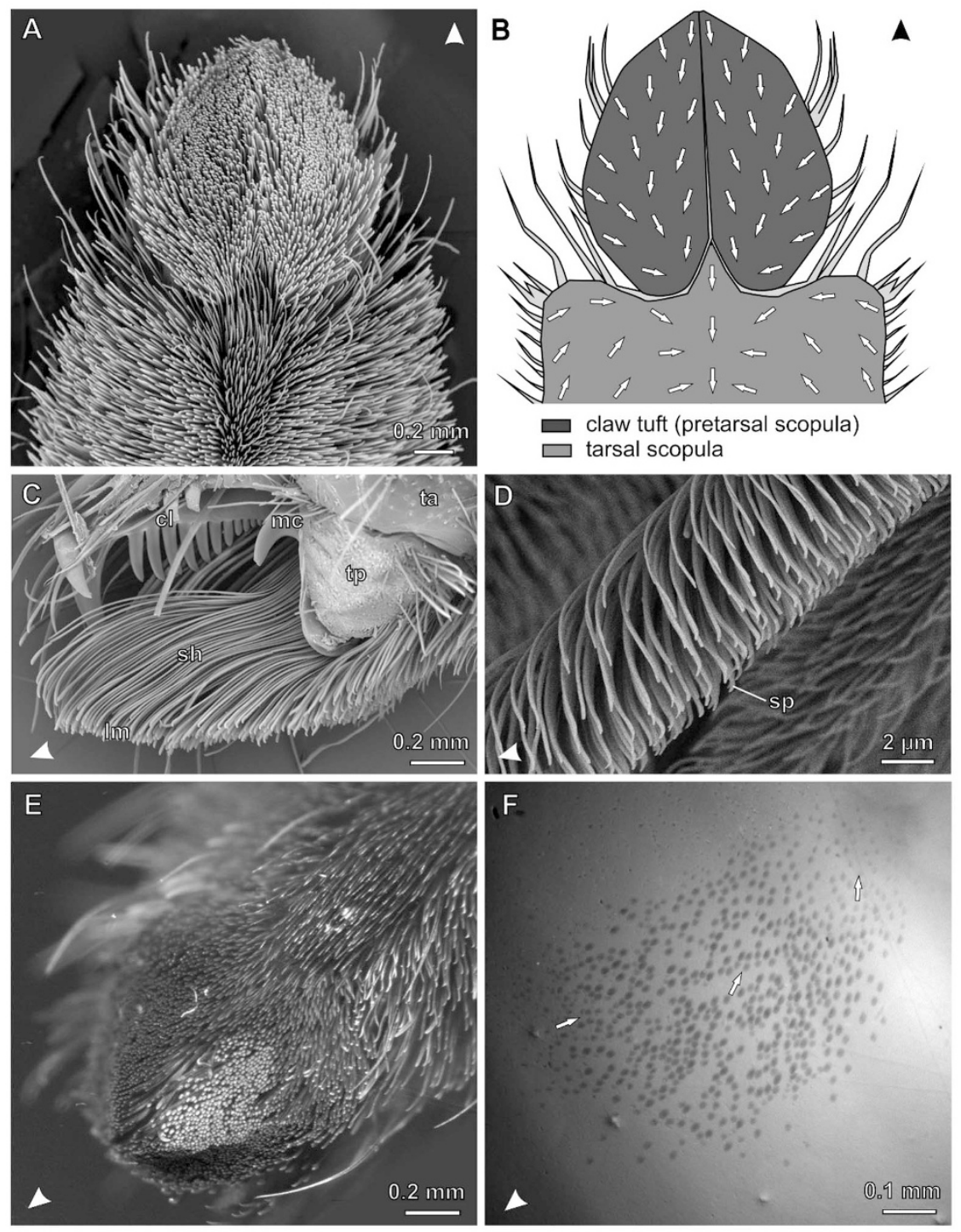

Figure $1 \mid$ The hairy attachment device of the wandering spider C. salei. Arrowheads indicate distal direction. (A) SEM micrograph of the distal L1 tarsus. (B) Diagram of the distal part of the tarsus illustrating different scopulae and the orientation of spatula-covered sides of setae (arrows). (C) SEM micrograph of the lateral view of L4 claw tuft, partly shaved. cl, tarsal claw; lm, distal lamellae of setae, bearing the the spatulate setules; mc, middle hook (reduced third claw); sh, seta shafts; ta, tarsus; tp, tenent pad (D) Lateral view of the distal part of a single seta in the median claw tuft. sp, spatula (E) L4 claw tuft of a living C. salei clinging upside-down on a Plexiglas Petri dish. Setae that are in contact are aligned in one plane and reflecting the light of the lateral LED light source at the same angle (bright spots). (F) Same with coaxial illumination (note slightly stronger magnification than in E). Contacting setae appear as darken oval spots, indicating that only the distal tips of setae are in real contact with Plexiglas. Arrows indicate the orientation of setae. 
be retracted by the claw levator muscle ${ }^{21}$. This was previously described for the Salticidae $e^{15,22}$. In contrast to the dionychan jumping spiders, where the claw tuft is hemispherically shaped ${ }^{5}$, it is tectiform in C. salei: The two parts of the claw tuft in C. salei form a planar surface each, which stand at an angle of $130^{\circ}$ to each other.

Total claw tuft area slightly increases from anterior to posterior legs: $0.93 \mathrm{~mm}^{2}$ on L1, $0.96 \mathrm{~mm}^{2}$ on $\mathrm{L} 2,0.99 \mathrm{~mm}^{2}$ on $\mathrm{L} 3$ and $1.16 \mathrm{~mm}^{2}$ on L4 (data previously published $\mathrm{in}^{5}$ ). Setal density is $2.4 \times 10^{-3} \mu \mathrm{m}^{-2}$, on average, and differs only marginally between the legs. Thus, the absolute number of adhesive setae increases from anterior to posterior leg claw tufts.

Friction properties. While sliding of the claw tuft, friction forces increased rapidly and remained constant after about $1.5 \mathrm{~s}$ from the beginning of the sliding motion with a constant velocity of $200 \mu \mathrm{m}$ per second (Fig. 2). In the fresh samples, friction coefficient was about two times higher in pulling direction (mean \pm sd: $\mu=1.09 \pm$ $0.19, \mathrm{~N}=43)$ than in pushing direction $(\mu=0.60 \pm 0.18, \mathrm{~N}=42$, Wilcoxon rank sum test: $\mathrm{p}<0.01$ ) (Fig. 3). In the air dried samples, the difference was even greater $(\mu=1.21 \pm 0.29, \mathrm{~N}=141$ in pulling; $\mu=0.43 \pm 0.16, \mathrm{~N}=141$ in pushing, Wilcoxon rank sum test: $\mathrm{p}<0.01$ ).

In pulling, frictional coefficients increased in dry claw tufts in the series from anterior to posterior legs (Fig. 4). It was about a half part higher in the claw tufts of $L 4(\mu=1.40 \pm 0.15, N=40)$ than that of L1 $(\mu=0.92 \pm 0.22, N=34)$. In L2 and L3 friction was in the medium range $(\mu=1.15 \pm 0.18, \mathrm{~N}=15$ and $\mu=1.27 \pm 0.25, \mathrm{~N}=52$, respectively). Differences between friction coefficients of legs were significant, except between L2 and L3 (Pair wise Wilcoxon rank sum test with Bonferroni correction: $\mathrm{p}<0.05)$. In pushing, frictional coefficients did differ significantly between the first three leg pairs (Pair wise Wilcoxon rank sum test with Bonferroni correction: $\mathrm{p}<0.05$ ), but there was no continuous increase in the series from anterior to

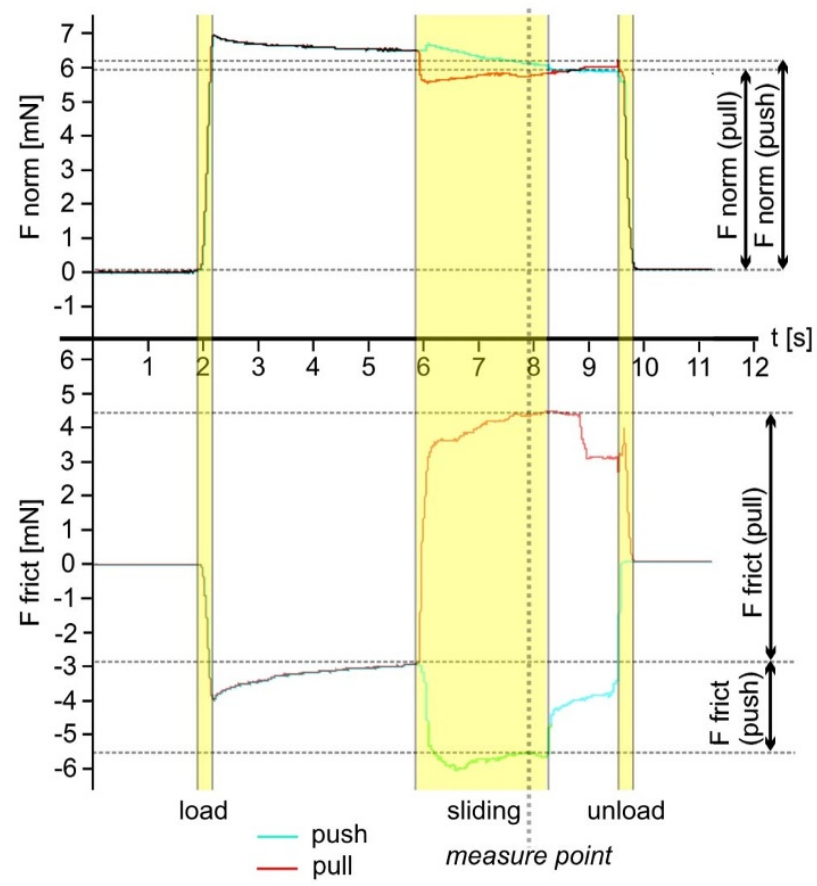

Figure $2 \mid$ Time-force curves of a typical experimental run for the pulling (red line) and the pushing (blue line) case. Normal forces (graph above) and friction forces (graph below) were recorded simultaneously. Values were obtained from forces recorded after two seconds after shear movement was started (measure point), to ensure that the contact between the pad and substrate was formed and friction forces have reached plateau. Friction coefficient $\mu$ was calculated as the quotient between friction and normal force.
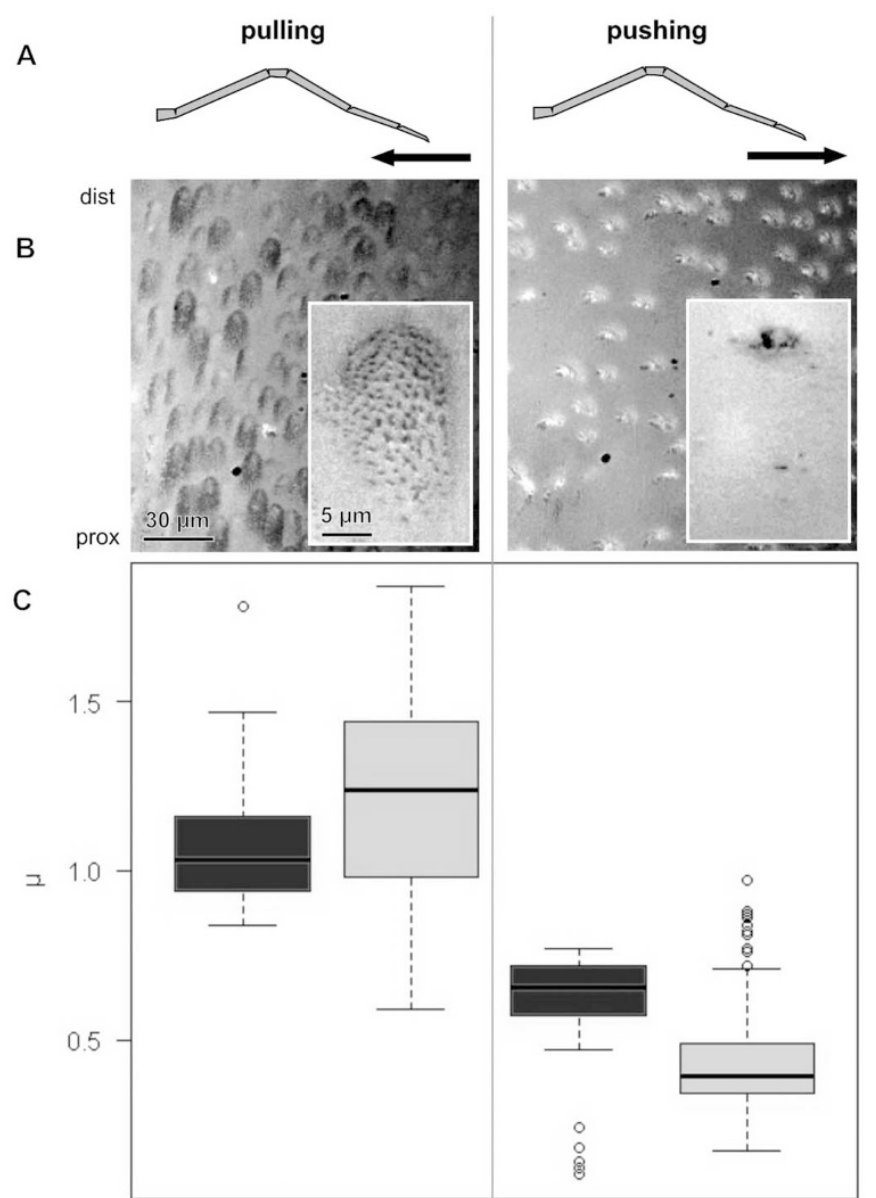

samples dry samples

Figure 3 Comparison of friction coefficients $\mu$ of sheared claw tufts between pulled and pushed conditions of the leg in fresh and air dried samples. Columns show data from shear direction as labelled above.

(A) Schematic illustration of the lateral view of the leg with an indication of shear direction. (B) RICM images of the distal part of the L4 claw tuft under the corresponding shear movement, indicating the difference in contact area (insets show single seta in contact). (C) Boxplot of friction coefficient $(\mu)$ data, illustrating median, interquartile range and extreme values.

posterior legs (L1: $\mu=0.41 \pm 0.09, \mathrm{~N}=34 ; \mathrm{L} 2: \mu=0.53 \pm 0.05, \mathrm{~N}=40$; L3: $\mu=0.38 \pm 0.05, N=52$ ). Friction coefficient of the pushed L4 did not differ significantly from those of the other legs, due to the strong variation of obtained data $(\mu=0.49 \pm 0.22, \mathrm{~N}=40)$.

Lateral shearing revealed a frictional anisotropy within both lobes of the divided claw tuft (Fig. 5). A retrolateral shift of the cover slip (forward movement of the leg simulated) induced low friction in the prolateral pad $(\mu=0.39 \pm 0.06, \mathrm{~N}=13)$ and high friction in the retrolateral pad $(\mu=1.25 \pm 0.16, N=8)$. For the prolateral shift of the coverslip (backwards movement of the leg simulated) the opposite effect was observed (prolateral pad: $\mu=1.25 \pm 0.16, \mathrm{~N}=13$; retrolateral pad: $\mu=0.44 \pm 0.06)$.

Visualisation of the contact behaviour. Microscopical observation with coaxial illumination of the claw tuft of living spider in contact with the Plexiglass revealed, that usually only one of both lobes of each claw tuft is in contact (Fig. 1E, F). Thus, during resting, only a small part of the whole claw tuft area is in contact.

The RICM study approach revealed, that spatulae get in contact, when claw tuft is sheared proximally, whereas the contact is broken, when it is sheared distally (Fig. 3, 6). Leg pulling caused a strong 

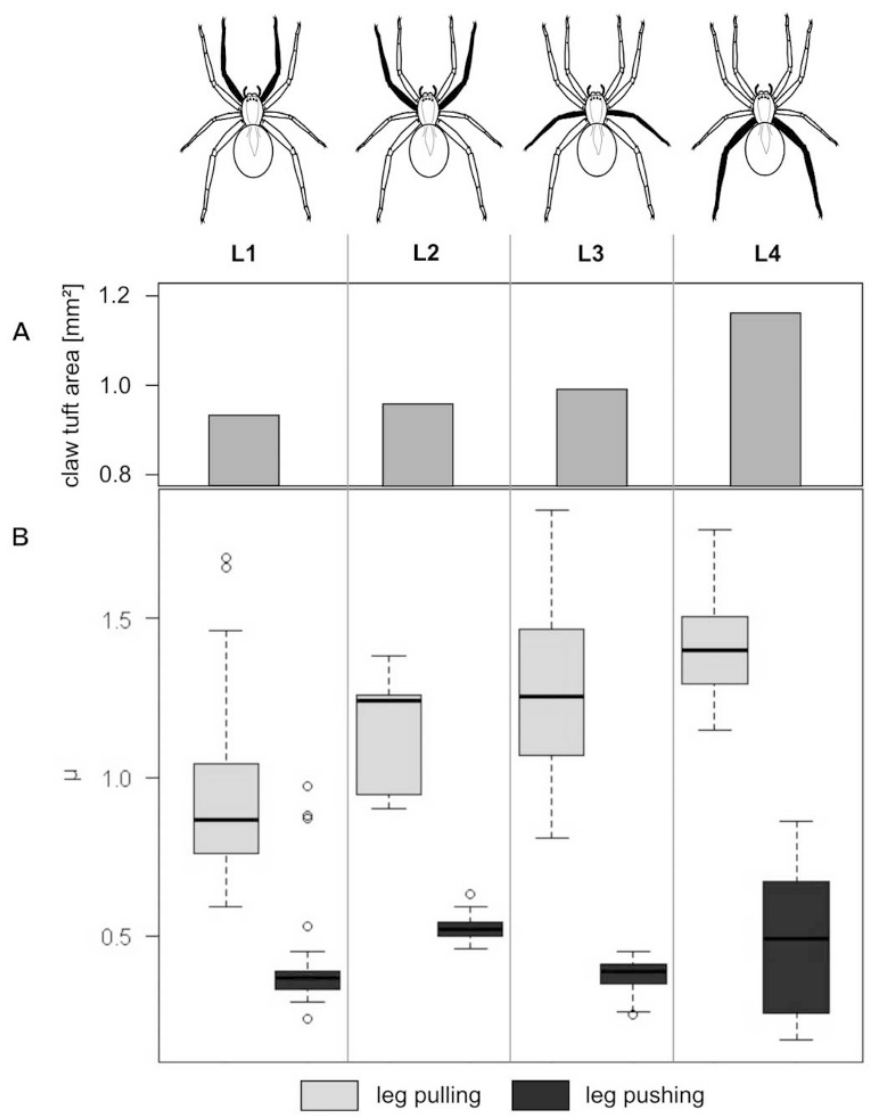

Figure 4 - Comparison of friction coefficients $\mu$ of sheared claw tufts of different leg pairs (L1-L4). (A) Barplot showing total claw tuft area of the corresponding legs. (B) Boxplot presenting friction coefficient data illustrating median, interquartile range and extreme values.

increase of the contact area and a proper alignment of setae/spatulae. Leg pushing induced turn over of the distal lamella of the seta and therefore a contact formation by the backside of distal setae. In this condition, contact area was much lower than at pulling, since spatulae are lacking on the backside of setae. At high magnifications ( $\times 630)$ spatulae could be identified as triangular dark spots on the bright background of the image. In the proximally moved seta, spatular contact points are regularly spread over the whole area of the setal tip (Fig. $3 \mathrm{~B}$, inset left). An inversion of the sliding direction induces a stepwise breaking of contacts of neighbouring spatulae along a crack propagating zone by the peel-off of the setal lamella. Crack propagation was mainly observed from proximal to distal parts of the seta. However, cracks sometimes also emerged from the centre or lateral side of the contacting setal part.

\section{Discussion}

A characteristic feature of hairy attachment devices in general is the anisotropy of frictional properties ${ }^{14,16,18,19,23}$. In spiders, this is based on the asymmetrical distribution of spatula-bearing setules on the tips of lamelliform setae ${ }^{14}$. In geckos, frictional anisotropy also relies on the asymmetrical distribution of the spatulae, but in contrast to spiders, where the setae are flexible to peel off at distal pushing, the gecko setae seem to be rather stiff and spatulae are detached by a change of the angle between individual seta and substrate ${ }^{16}$. In insects, where usually no branching of setae is present, anisotropy is explained by (1) the sloped configuration of setae, (2) the subdivision of the segmented tarsus leading to the peel-off of spatulae when pushing the leg, and (3) peeling off of individual spatulae ${ }^{18,24}$. Although mechanisms are slightly different in different animals,

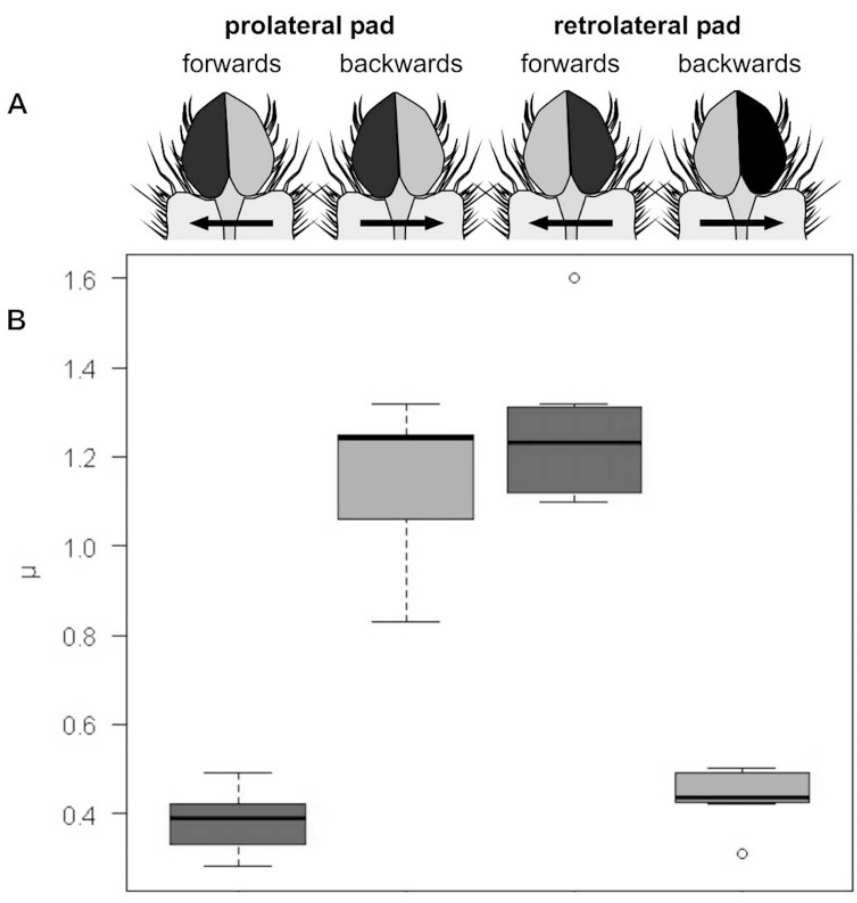

Figure 5 Comparison of friction coefficients $\boldsymbol{\mu}$ of laterally sheared individual lobes of bilobed claw tufts. (A) Schematic illustration of the direction of shearing of corresponding lobes, ventral view. Black areas mark the contacting lobe. Arrows indicate simulated slide direction of the tarsus. (B) Boxplot of friction coefficient data, illustrating median, interquartile range and extreme values.

the vector of highest friction force is generally directed away from the body and pads are activated through proximally directed shear forces. However, for the tarsal and metatarsal scopula of C. salei and Aphonopelma seemanni, contrary results were obtained ${ }^{14}$ and explained by the opposite arrangement of spatulae on the surface of individual setae in those leg regions.

The microscopical study of the claw tuft in Cupiennius salei revealed that its surface is not planar. This also holds for claw tufts of other spiders ${ }^{5,25}$. As the orientation of setae gradually changes within the claw tuft in both distal and lateral directions, the particular part of the tuft that is brought in contact mainly influences the direction of maximal friction. Our observations of living animals showed that only a small part of the claw tuft is in contact at the same time. This has also been observed in jumping spiders ${ }^{25}$. One may assume that spiders can control their attachment by the way how they stretch, rotate and place their legs. Furthermore, spiders must apply shear forces to get spatulae in contact and gain high friction with the substrate, which is important to attach properly. This is consistent with recent theoretical models on contact mechanics of spatulae ${ }^{26}$. Furthermore this may lead to a high increase of adhesion forces due to the application of small peel angles ${ }^{27}$. This is especially important for upside-down locomotion of the spider. Likewise shear forces applied in the opposite direction lead to a drop of frictional forces due to contact breakage by peeling-off facilitating detachment. This indicates that the attachment of the spider to the ceiling must strongly depend on the muscle activity that continuously generates shear forces along the substrate, as it was recently shown for stick insects ${ }^{28}$.

Pulling leads to an increased angle between tarsus and substrate, thus only the claw tuft is in contact. If the leg is outstretched, the angle decreases and parts of the tarsal scopula might get in contact. Because the direction of the highest friction in tarsus and metatarsus opposes the one in the claw tuft, both these parts can generate the necessary friction for forward thrust, when leg is pushed. This indicates that on 

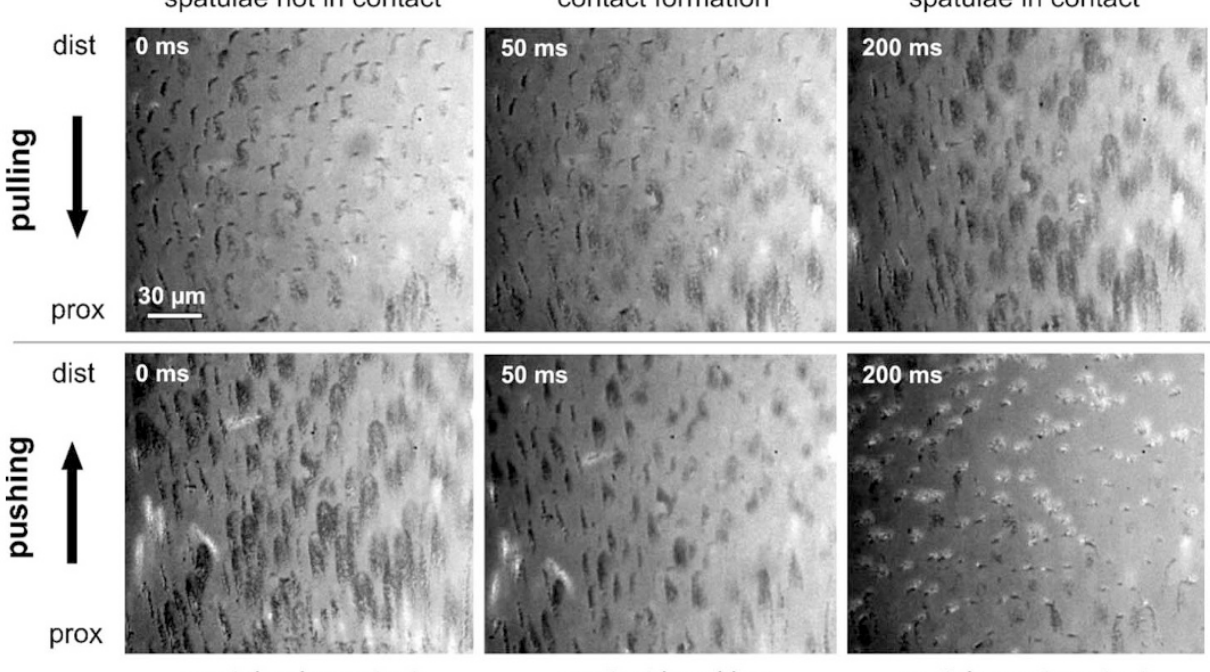

spatulae in contact

contact breaking

spatulae not contact

Figure $6 \mid$ RICM sequence of individual images of the sheared setae in the distal part of L4. Arrows indicate shear direction of the claw tuft; prox, proximal; dist, distal.

smooth surfaces, pulling with anterior legs might be more important for moving forwards than pushing by posterior legs. Tarsal scopula might be more important in the vertical resting position of the spider. Pulling with the anterior legs, while resting head-down, can not increase friction, and that is why we assume the key role of pulling by posterior legs in such a behavioural situation. Here, anterior legs are outstretched and tarsal scopula is presumably in contact generating friction by pushing. Together with an additional support by the dragline, such leg configuration may cause a strong attachment on smooth walls (Fig. 7).

Tarsal and metatarsal scopulae might also play a role in prey grasping and manipulation, like it has been reported from other lycosoids $s^{29}$ as well as salticids ${ }^{30}$ and palpimanids ${ }^{31}$. However, in $C$. salei metatarsal scopula includes long spines that erect with increasing hemolymph pressure (personal observation) presumably during prey capture. Thus, the prey may rather contact erected spines than scopulae, and the role of the latter in the prey-capture needs special attention in the future.

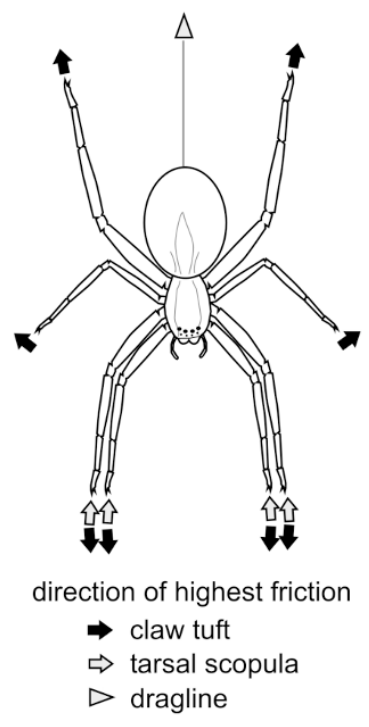

Figure $7 \mid$ Directions of highest friction in the vertically resting position of $C$. salei.
The results of the force measurements suggest that friction forces play the major role in the attachment of spiders. The specific arrangement of claw tufts at the distal tips of the eight outstretched walking legs is essential for strong attachment even on smooth glass surfaces: The direction of highest friction in each claw tuft is directed away from the mass centre of the animal. Due to the radial arrangement of legs, there is always a frictional counterpart for each claw tuft. Thus, the whole animal works like a holdfast. This has previously been hypothesized by Hill ${ }^{22}$. Because high friction can also be achieved by lateral shear forces in the proximal and lateral parts of claw tuft, holdfast-like mechanism can even work between neighbouring legs.

Our study showed that attachment forces are not linearly proportional to the number of attaching legs, but show an exponential increase $\mathrm{e}^{32}$. This indicates that attachment forces depend on the interaction between legs and are not dependent on the apparent contact area alone. We observed that at least two leg pairs in Cupiennius salei are necessary to attach to a glass surface ${ }^{32}$. However, in this experiment whole leg pairs were immobilized stepwise from posterior to anterior and in the opposite. Thus, the directly opposing legs were not left intact. Presumably, the spider would even be able to attach with one anterior and one posterior leg of the other body side.

Radial arrangement of the frictional units is also described from other hairy attachment devices like single gecko feet ${ }^{16}$. Similar effects were recently observed in beetles ${ }^{19}$. Direction-dependence was found for hairy as well as smooth adhesive pads in insects ${ }^{18}$. Thus, this seems to be a general principle and an optimal solution for generating strong attachment forces and providing fast and effortless detachment at the same time.

Spider scopula has been reported to be a dry adhesive throughout literature ${ }^{6}$, although capillary effects of a natural water film on substrates may play an important role ${ }^{8,13}$. However, Peattie et al. reported that spiders occasionally secrete some fluid in the contacts of their attachment pads ${ }^{10}$. In mygalomorph spiders thread-like traces left on the substrate by scopulae were demonstrated ${ }^{9,33}$. Our results show, that drying of claw tuft specimens does not lead to a drop in friction force. Thus, foot secretions seem to be not indispensable for a proper attachment. A close inspection of spatulae did not reveal the presence of any pores, where fluids could be delivered to the surface. The presence of observed tarsal fluids in spiders was recently explained by secretions of chemosensitive setae ${ }^{34}$. These might serve as a solvent for signal molecules and might thus occur, when spider collects chemical information about the substrate, to which it is attaching. 


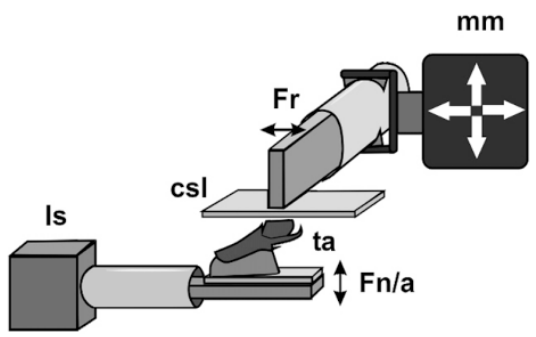

Figure $8 \mid$ Experimental setup for friction force measurements. Csl, cover slip; Fn/a, force sensor deflecting vertically, recording normal force and adhesive force; Fr, force sensor deflecting horizontally, recording friction force; ls, laboratory stand; $\mathrm{mm}$, micromanipulator; ta, tarsus with upwards pointing claw tuft attached to the force sensor.

\section{Methods}

Animals and behavioural observations. Three living individuals of the hunting spider Cupiennius salei KEYSERLING 1877 (Ctenidae) were obtained from a laboratory stock of the Department of Neurobiology, University of Vienna, Austria. Spiders were kept in cylindrical glasses at the room temperature and 95\% relative humidity and fed with house crickets (Acheta domestica) obtained from the local pet shop.

The claw tufts were observed with aid of a stereo microscope (M205 A, Leica Microsystems, Wetzlar, Germany) under lateral and coaxial illumination in spiders resting upside-down on the smooth transparent surface of Plexiglas Petri dishes.

Scanning electron microscopy (SEM). Tarsi of the four pairs of walking legs of one body side were ablated with a scalpel in spiders anaesthetized with carbon dioxide. The samples were air dried, mounted on metal stubs and sputter coated with a $15 \mathrm{~nm}$ layer of gold-palladium. Samples were viewed in the SEM TM-3000 (Hitachi Ltd., Tokyo, Japan) at $15.0 \mathrm{kV}$ using back-scattered electron (BSE) detector.

Force measurements. The setup for force measurements was as previously described by Niederegger and Gorb ${ }^{14}$ and is displayed in Fig. 8. Freshly ablated tarsi of different walking legs from spiders anaesthetized with carbon dioxide were shaved at their dorsal side and mounted on a Plexiglas slide with bees wax. Tarsi were positioned in the wax so that the median surface of the setal array of the claw tuft was parallel to the Plexiglas slide. Those samples were attached with double sided adhesive tape to the distal cantilever of a load cell force transducer with $10 \mathrm{~g}$ force range (World Precision Instruments Inc., Sarasota, FL, USA). A second force transducer of the same type was attached to a micromanipulator (DC3001R with controller MS314, World Precision Instruments Inc., Sarasota, FL, USA) and placed perpendicularly to the first one. A clean glass cover slip was mounted on the lateral edge of the cantilever. Thus, normal force and friction force could be recorded simultaneously. Force curves were recorded with AcqKnowledge 3.7.0 software (Biopac Systems Ltd, Goleta, CA, USA). A laterally installed stereo microscope was used to monitor the sample movements and the proper contact formation between the claw tuft and smooth substrate.

Experiments were performed at an environmental temperature of $20-23^{\circ} \mathrm{C}$ and a relative humidity of $20-25 \%$. The cover slip was brought into contact with the claw tuft and loaded until normal force reached about $7 \mathrm{mN}$. Then it was horizontally moved for $3 \mathrm{~s}$ with the constant velocity of $200 \mu \mathrm{m} \cdot \mathrm{s}^{-1}$ in the proximal (simulating leg pushing) and distal (simulating leg pulling) direction, and the friction forces, resisting these movements, was recorded. Proximal and distal sliding experiments were done in a randomized order.

Similar force measurements were repeated with the same but air dried samples after two days. Additionally, pro- and retrolateral shearing experiments were performed in the pro- and retrolateral lobes of the claw tuft on an air dried anterior leg tarsus. For this purpose, the leg sample was positioned in the way that the surface of respective lobe was oriented parallelly to the surface of the glass cover slip.

Force data were obtained by respective processing of the recorded time-force curves. We have taken into account values recorded after two seconds after shear movement was started, to ensure that the contact between the pad and substrate was formed and friction forces have reached plateau. Friction coefficient $\mu$ was calculated as the quotient between friction and normal force. Data were statistically compared with R software package (version 2.13.2, http://www.r-project.org).

Reflection interference contrast microscopy (RICM). Contact behaviour between tuft pad and glass substrate was visualized with an inverted light microscope (Axio Observer.A1, Carl Zeiss Microscopy GmbH, Göttingen, Germany). In the RICM mode, the light source is positioned in a way that light is reflected at the interface of direct (real) contact between the glass slide and the object. Zones of direct contact appear as dark spots on the bright background. Similar visualisation techniques were previously used in studies of attachment of cells ${ }^{35}$ and frogs ${ }^{36}$.

A cleaned glass cover slip was mounted on the stage and viewed at $\times 200-630$ (oil immersion) magnification. The air dried claw tuft was glued onto a sample holder and positioned with the ventral side onto the cover slip. The stage was then manually moved vertically and laterally and the behaviour of spatulae in contact with glass was recorded with a high speed video camera (Photron Fastcam SA1.1, VKT Video Kommunikation GmbH, Pfullingen, Germany) at 500-1000 frames per second.

1. Varenberg, M., Pugno, N. M. \& Gorb, S. N. Spatulate structures in biological fibrillar adhesion. Soft Matter 6, 3269-3272 (2010).

2. Gorb, S. N. Attachment devices of insect cuticle. Kluwer Academic Publishers, Dordrecht (2001).

3. Federle, W. Why are so many adhesive pads hairy? J. Exp. Biol. 209, 2611-2621 (2006).

4. Autumn, K. \& Gravish, N. Gecko adhesion: evolutionary nanotechnology. Phil. Trans. R. Soc. A 366, 1575-1590 (2008).

5. Wolff, J. O. \& Gorb, S. N. Comparative morphology of pretarsal scopulae in eleven spider families. Arthropod Struct. Dev. 41, 419-433 (2012).

6. Roscoe, D. T. \& Walker, G. The adhesion of spiders to smooth surfaces. Bull. Br. Arachnol. Soc. 8, 224-226 (1991).

7. Kesel, A. B., Martin, A. \& Seidl, T. Adhesion measurements on the attachment devices of the jumping spider Evarcha arcuata. J. Exp. Biol. 206, 2733-2738 (2003).

8. Wolff, J. O. \& Gorb, S. N. The influence of humidity on the attachment ability of the spider Philodromus dispar (Araneae, Philodromidae). Proc. R. Soc. B 279, 139-143 (2012).

9. Gorb, S. N. et al. Silk-like secretion from tarantula feet. Nature 443, 407 (2006).

10. Peattie, A. M., Dirks, J. H., Henriques, S. \& Federle, W. Arachnids secrete a fluid over their adhesive pads. PLoS ONE 6, e20485 (2011).

11. Coddington, J. A. \& Levi, H. W. Systematics and evolution of spiders. Ann. Rev. Ecol. Syst. 22, 565-592 (1991).

12. Jocqué, R. \& Dippenaar-Schoeman, A. S. Spider Families of the World. $2^{\text {nd }}$ edition. Royal Museum for Central Africa, Tervuren, Belgium (2007).

13. Homann, H. Haften Spinnen an einer Wasserhaut? Naturwissenschaften 44, 318 319 (1957).

14. Niederegger, S. \& Gorb, S. N. Friction and adhesion in the tarsal and metatarsal scopulae of spiders. J. Comp. Physiol. A 192, 1223-1232 (2006).

15. Hill, D. E. The pretarsus of salticid spiders. Zool. J. Linn. Soc. 60, 319-338 (1977).

16. Autumn, K., Dittmore, A., Santos, D., Spenko, M. \& Cutkosky, M. Frictional adhesion: a new angle on gecko attachment. J. Exp. Biol. 209, 3569-3579 (2006).

17. Gorb, S. N. \& Scherge, M. Biological microtribology: Anisotropy in frictional forces of orthopteran attachment pads reflects the ultrastructure of a highly deformable material. Proc. Roy. Soc. London B, 267, 1239-1244 (2000).

18. Bullock, J. M. R., Drechsler, P. \& Federle, W. Comparison of smooth and hairy attachment pads in insects: friction, adhesion and mechanisms for directiondependence. J. Exp. Biol. 211, 3333-3343 (2008).

19. Gorb, S. N. Biological fibrillar adhesives: Functional principles and biomimetic applications. In: da Silva, L. F. M., Öchsner, A., Adams, R. D., (eds.), Handbook of adhesion technology. Springer-Verlag Berlin Heidelberg, pp. 1409-1436 (2011).

20. Foelix, R. F. \& Chu-Wang, I.-W. The structure of scopula hairs in spiders. Proc. $6^{\text {th }}$ Int. Arachnol. Congr. 1974, Free University Amsterdam, pp. 156-157 (1975).

21. Speck, J. \& Barth, F. G. Vibration sensitivity of pretarsal slit sensilla in the spider leg. J. Comp. Physiol. A 148, 187-194 (1982).

22. Hill, D. E. The pretarsus of salticid spiders. Version 3. Peckhamia Epublications141 (2006).

23. Creton, C. \& Gorb, S. N. Sticky Feet: From Animals to Materials. MRS Bull. 32 (2007).

24. Niederegger, S. \& Gorb, S. N. Tarsal movements in flies during leg attachment and detachmenton a smooth substrate. J. Insect Physiol. 49, 611-620 (2003).

25. Hill, D. E. Function of the pretarsus in living Phidippus regius. Peckhamia 1, 70-71 (1978).

26. Filippov, A., Popov, V. L. \& Gorb, S. N. Shear induced adhesion: Contact mechanics of biological spatula-like attachment devices. J. Theoretical Biol. 276, 126-131 (2011).

27. Pesika, N. S., Tian, Y., Zhao, B., Rosenberg, K., Zeng, H., McGuiggan, P., Autumn, K. \& Israelachvili, J. N. Peel-Zone Model of Tape Peeling Based on the Gecko Adhesive System. J. Adh. 83, 383-401 (2007).

28. Bußhardt, P., Gorb, S. N. \& Wolf, H. Activity of the claw retractor muscle in stick insects in wall and ceiling situations. J. Exp. Biol. 214, 1676-1684 (2011).

29. Rovner, J. S. Adhesive hairs in spiders: behavioral functions and hydraulically mediated movement. Symp. Zool. Soc. Lond. 42, 99-108 (1978).

30. Foelix, R. F., Jackson, R. R., Henksmeyer, A. \& Hallas, S. Tarsal hairs specialized for prey capture in the salticid Portia. Rev. Arachnol. 5, 329-334 (1984).

31. Pekár, S., Šobotník, J. \& Lubin, Y. Armoured spiderman: morphological and behavioural adaptations of a specialised araneophagous predator (Araneae: Palpimanidae). Naturwissenschaften 98, 593-603 (2011).

32. Wohlfart, E., Wolff, J. O., Arzt, E. \& Gorb, S. N. Two together is more than a sum of two separate singles: collective effect of attachment organs in the spider Cupiennius salei Keyserling (Arachnida, Ctenidae). Submitted.

33. Rind, F. C., Birkett, C. L., Duncan, B.-J. A. \& Ranken, A. J. Tarantulas cling to smooth vertical surfaces by secreting silk from their feet. J. Exp. Biol. 214 1874-1879 (2011).

34. Foelix, R. F., Rast, B. \& Peattie, A. M. Silk secretion from tarantula feet revisited: alleged spigots are probably chemoreceptors. J. Exp. Biol. 215, 1084-1089 (2012). 
35. Michaelis, S., Robelek, R. \& Wegener, J. Studying cell-surface interactions in vitro: A survey of experimental approaches and techniques. Adv. Biochem. Engin. /Biotechnol. 126, 33-66 (2012).

36. Federle, W., Barnes, W. J. P., Baumgartner, W., Drechsler, P. \& Smith, J. M. Wet but not slippery: boundary friction in tree frog adhesive toe pads. J. R. Soc. Interface 3, 689-697 (2006).

\section{Acknowledgements}

We thank Dr. Clemens Schaber and Prof. Dr. Friedrich G. Barth (Department of Neurobiology, University of Vienna, Austria) for providing experimental animals and Dr. Jan Michels for support in microscope techniques. This work was supported by the German Science Foundation (DFG) Initiative "Bionik" (DFG grant GO995/7-1 to SG).

\section{Author contributions}

Wolff and Gorb designed the experiment; Wolff performed the experiment and wrote the manuscript; Gorb reviewed the manuscript.

\section{Additional information}

Competing financial interests: The authors declare no competing financial interests.

License: This work is licensed under a Creative Commons

Attribution-NonCommercial-ShareALike 3.0 Unported License. To view a copy of this license, visit http://creativecommons.org/licenses/by-nc-sa/3.0/

How to cite this article: Wolff, J.O. \& Gorb, S.N. Radial arrangement of Janus-like setae permits friction control in spiders. Sci. Rep. 3, 1101; DOI:10.1038/srep01101 (2013). 